\title{
'Out-of-the-box' thinking to help stop the spread of antibiotic resistance
}

\author{
"What is important is that all possible solutions relating to the fight against antibiotic \\ resistance should be open for discussion."
}

John P Hays*

First draft submitted: 19 June 2016; Accepted for publication: 8 July 2016;

Published online: 29 July 2016

\begin{abstract}
Antibiotic resistance is a major global problem and many schemes and guidelines have been established to help reduce its spread [1]. In this respect, so-called 'out-of-the-box' thinking may play a role in effectively educating and 'nudging' the behavior of physicians and the general public toward actions that will help reduce the spread of current and future antibiotic resistance. The following 'out-of-the-box' suggestions may appear controversial, cynical or unworkable at the current moment in time, but if antibiotic resistance continues to spread at its current rate, we may need to rethink current policies and seriously consider adopting alternative messages and technologies to help fight this worldwide endemic.
\end{abstract}

\section{The cult of celebrity}

In modern society, the general public places much attention and admiration on famous celebrities and on the actual pursuit of celebrity per se (think of Hollywood or Bollywood film stars and TV programs such as The X Factor, Britain's Got Talent, etc.). Celebrities could be employed to generate so-called 'infomercials', informing the general public about the threat of global antibiotic resistance and urging the responsible use of antibiotics. More likely to capture the public's imagination is a true story, especially a 'battle won' under extreme circumstances. In this respect, celebrities could explain their own personnel or family experience with infectious diseases, and how the use of antibiotics helped (or did not help in the case of extreme antibiotic resistance) cure these infections [1]. This approach may appear cynical, but it could potentially be very effective in channeling the public's attention to the problems associated with antibiotic resistance. Importantly, celebrities could be chosen to reflect national or regional 'celebrity status' in order to generate maximum impact within different communities, belief systems and geographies.

\section{'Stop \& go traffic lights' for food production}

The use of antibiotics in food production may be a major contributor to the development of antibiotic resistance in animals and possibly in humans. Perhaps we could use economics and the potential buying power of the general public to 'nudge' food producers to produce animal-based foods that have not been produced using antibiotics? Additionally, it is not unreasonable to think that consumers would 'vote with their pockets' and pay for slightly more expensive food products when presented with food packaging labels stating 'antibioticfree production' or 'reduced antibiotic use'. This extra 'premium' could then be given back to food producers to encourage them to limit their use of antibiotics within the agricultural sector. Alternatively, a 'traffic-light' system (based on for example: the actual use

*Department of Medical Microbiology \& Infectious Diseases, Erasmus University Medical Centre Rotterdam (Erasmus MC),

\section{KEYWORDS}

- antibiotic resistance $\bullet$ ideas

- out-of-the-box 


"One of the major
problems with the spread
of antibiotic resistance is
that patients tend to keep
hold of any unused
antibiotic tablets for
possible unprescribed
future use..."

future use...” of antibiotics during food production; or the use of antibiotics of 'last resort', e.g., colistin; or on the level of use of antibiotics within a particular agricultural region), could bring the problem of antibiotic resistance into the full view of the general public every time that they do their 'weekly shopping'.

\section{Watson \& homes}

Cognitive (the act of knowing or perceiving) healthcare may be 'just around the corner' and many different fields of medical science could be impacted by the development of supercomputer algorithms and 'fuzzy logic'. One such supercomputer that has gained much attention is IBM's 'Watson' [2], a system developed as part of IBM's DeepQA project that can read 40 million documents in $15 \mathrm{~s}$ [3]. One of the medical fields currently under investigation by Watson is in the diagnosis of cancer [4]. In the future, supercomputer algorithms produced by Watson may be utilized by physicians or individuals to help guide them in their lifestyle choice and medical decisions. Data collection could involve linking local healthcare data, for example, local antibiotic prescribing practices, local antibiotic resistance profiles, rates of patient relapse, etc., to 'wearable technologies' [5] measuring, for example, fever, increased pulse rate, change in blood pressure and rate of sweat production, among others. Algorithms may then be able to distinguish, for example, between a viral and a bacterial infection. If an algorithm replies "There is a $90 \%$ probability that you do not require antibiotics," then the individual may be less inclined to visit their family doctor. Real-time monitoring allows extra comments such as "I will keep monitoring your situation and inform you when there is more than $5 \%$ difference in the expected probability." Governments and healthcare authorities could be encouraging companies such as IBM, Apple, Google, etc., to think about anonymized data collection and the development of supercomputer algorithms for global antibiotic resistance.

\section{Deposits \& reimbursements on antibiotic tablets}

One of the major problems with the spread of antibiotic resistance is that patients tend to keep hold of any unused antibiotic tablets for possible unprescribed future use, which is in part also due to the size of antibiotic packaging (steps are already being undertaken to reduce the size of packets of antibiotics). Perhaps a deposit/reimbursement system could be introduced whereby the patient pays a small deposit when they receive a prescription for antibiotics? The patient would then be reimbursed for every unused tablet returned to a pharmacist or a family doctor. The actual amount of deposit would be dependent on the prescribed number of antibiotic tablets to be taken, as prescribed by a physician, and the extra number of tablets available in the antibiotic packets prescribed. If patients are serious about being cured, they will take the whole course of tablets as prescribed and be less inclined to worry about the small deposit that they have paid. Patients should be requested to hand in the original box (containing the 'Use By' date), and the original prescription, along with the original tablets. Of course, there will always be the risk that fake tablets will be produced and handed in for reimbursement and there will be a balance necessary between physicians prescribing too many antibiotic tablets and the financial incentive (the reimbursement price per tablet).

\section{Multivitamins first!}

Frequently, patients presenting with symptoms of infectious disease visit their family doctor expecting to receive antibiotics. Patients may then be offended if no antibiotic is prescribed, as the actual act of prescribing a tablet often indicates to the patient that the doctor is listening and taking the concerns of the patient seriously. So, instead of prescribing antibiotics that are not necessary, or no antibiotics at all, perhaps physicians should prescribe multivitamin tablets, while explaining that 'antibiotics are not necessary', but that 'the patient may require extra help to enable their bodies to recover from the infection'? Compared with the prescribing of unnecessary antibiotics, the prescribing of multivitamins is unlikely to generate harm, would be low cost and could potentially help the patient recover from their illness. Importantly, psychologically speaking, the patient would feel that the physician is taking their complaint seriously. Additionally, prescribing multivitamins circumvents the possible negative connotations associated with the prescribing of analgesics or placebos (as vitamin substitutes) to patients, and at the same time would help reduce antibiotic prescribing and maintain patient confidence in their physicians. 


\section{Antibiotics as cigarettes}

If the overuse of antibiotics can lead to antibiotic resistance and death, then perhaps antibiotics should be regarded in the same light as cigarettes? Similarities between cigarette smoking and taking antibiotics include: the general public may ignore the advice of health professionals relating to both subjects; patients may consider that any negative effects will only occur to someone else, or will occur sometime in the future, or may believe that taking one small dose now will have little effect on the development of serious side effects to other individuals (think of passive smoking and the transfer of antibiotic resistant organisms between individuals). Of course, smoking is physically addictive and taking antibiotics is not - though the reassurance obtained and possible feeling of 'well-being' associated with taking antibiotics to cure an infection may generate at least a 'meaning response' [6] in the mind of the patient. If we accept healthcare parallels between antibiotic use and cigarette smoking, then perhaps we should consider introducing 'health warnings' on packets of antibiotics? For example, texts such as "In the future, antibiotic resistance may kill 1 person every 3 seconds!" or "Please use antibiotics sparingly and only when prescribed by a physician" [7]. Striking images could include a picture of a particularly mucoid stain of Klebsiella pneumoniae [8], or a generic picture of a sick patient lying in a hospital bed. In order to increase their impact, these images and warnings should be adapted to fit with local languages, ethnicities and cultures.

\section{Ban or levy generic antibiotics without prescription}

A generic medicine is a medicine which is equivalent to an existing pharmaceutical medicine (similar administration route, dose, strength and quality), which is not sold under the original brand name, and are usually cheaper than the original brand-name medicine on which they are based. Further, generic medicines are sold nationally according to the regulations of individual governments [9]. The use of generic medicines (vaccines, drugs, etc.) may facilitate extended access to effective medicines and healthcare to poorer regions of the world, and in so doing, saves many lives. However, with specific respect to antibiotics, the purchase price of antibiotics is a major factor in driving sales, and the availability of cheap antibiotics for sale without a physician's prescription is one of the main drivers of increasing antibiotic resistance [10]. Perhaps then, governments should not only think about restricting the availability of antibiotics without prescription, but should at the same time place a financial levy on (or even attempt to ban outright) the sale of generic antibiotics available without prescription? Of course, in the age of international internet commerce, banning (the import of) generic antibiotics may be impossible to enforce [11]. However, online buyers of illegal 'generic antibiotics' without national levies [12] should be reminded that there is no guarantee regarding the quality of the product that they are buying and that they could be simply wasting their money compared with the standard of quality obtained by purchasing a prescribed antibiotic from a 'professional and respectable' source, such as a physician.

In this article, the author has presented seven 'out-of-the-box' suggestions for discussion and debate as part of the global effort to educate and bring behavioral change to the fight against antibiotic resistance. Readers may disagree with these suggestions or may even have their own 'out-of-the-box' suggestions. What is important is that all possible solutions relating to the fight against antibiotic resistance should be open for discussion.

\section{Financial \& competing interests disclosure}

This work has received funding from the European Union's Seventh Framework Programme for Health under grant agreement number 602860 (TAILOREDTreatment; www.tailored-treatment.eu). The author has no other relevant affiliations or financial involvement with any organization or entity with a financial interest in or financial conflict with the subject matter or materials discussed in the manuscript apart from those disclosed.

No writing assistance was utilized in the production of this manuscript.

\section{Open access}

This work is licensed under the AttributionNonCommercial-NoDerivatives 4.0 Unported License. To view a copy of this license, visit http://creativecommons.org/ licenses/by-nc-nd/4.0/ 


\section{OPINION Hays}

\section{References}

1 Review on Antimicrobial Resistance. Publications.

http://amr-review.org/Publications

2 IBM Watson Health www.ibm.com/smarterplanet/us/en/ ibmwatson

3 The DeepQA Project. www.research.ibm.com/deepqa/deepqa. shtml

4 IBM Watson for Oncology. www.ibm.com/smarterplanet/us/en/ ibmwatson/
5 Wearable technology. https://en.wikipedia.org/wiki/Wearable technology

6 Moerman DE, Jonas WB. Deconstructing the placebo effect and finding the meaning response. Ann. Intern. Med. 136(60), 471-476 (2002).

7 Review on Antimicrobial Resistance. Tackling drug-resistant infections globally: final report and recommendations (2016).

http://amr-review.org/sites/default/filesl

8 Kumabe A, Kenzaka T. String test of hypervirulent Klebsiella pneumonia. Q. J. Med. 107, 1053 (2014).

http://qjmed.oxfordjournals.org/content/107/
9 Wikipedia: generic drug. https://en.wikipedia.org/wiki/Generic_drug

10 Babu V, Suma C. Antibiotic pricing: when cheaper may not be better. Clin. Infect. Dis. 43(8), 1085-1086 (2006).

11 Toutain PL, Bousquet-Melou A. The consequences of generic marketing on antibiotic consumption and the spread of microbial resistance: the need for new antibiotics. J. Vet. Pharmacol. Therap. 36 , 420-424 (2013).

12 Online Pharmacy. Antibiotics. http://healthydancer.com/catalog/ Antibiotics.htm 